\title{
Optical and Electrochemical Properties of Silver-doped Yttrium Barium Copper Oxide as High-Temperature Superconducting Materials
}

\author{
Ping Gao \\ Department of Physics, Lvliang University, Lvliang, Shanxi, 033000, China \\ E-mail: pinggao@protonmail.com
}

doi: $10.20964 / 2021.06 .32$

Received: 22 March 2021 / Accepted: 6 April 2021 / Published: 30 April 2021

\begin{abstract}
This work focused on electrochemical and optical properties of yttrium barium copper oxide (YBCO) and Ag-doped YBCO (Ag-YBCO) as superconducting films. The films were synthesized through conventional solid state reactions. Morphology, crystal and chemical structure, optical and electrochemical properties of synthesized films were studied at room temperature. Morphological results using FESEM analysis showed that YBCO and Ag-YBCO films surface were covered with rectangular crystal grains which grew parallel and perpendicular to the basal plane. Studying the crystal structure using XRD analysis showed that there was the orthogonal superconducting phase of Y123 before sintering processing. XRD results also showed elimination of $\mathrm{BaCO}_{3}$ phase and formation of crystalline phase of $\mathrm{Ba}-\mathrm{Cu}-\mathrm{O}_{3}$ in sintered $\mathrm{YBCO}$ film and incorporation of fcc phase of Ag into the doped film. Raman analysis demonstrated the formation of oxide phases in YBCO and Ag-YBCO films. Optical analysis illustrated the formation of $\mathrm{AgO}$ in doped film could provide reactive atomic oxygen to the growth YBCO thin film. Study of electrochemical properties indicated that area specific resistance of $\mathrm{Ag}-\mathrm{YBCO}$ was lower than YBCO electrode and electrochemical response of Ag-YBCO was more stable than YBCO response because of more electrochemical activity of silver particles of doped film in aqueous solution. Furthermore, Ag addition in YBCO film can change in the charge carrier and electronic structure in the compound due to the formation of oxygen vacancies in the $\mathrm{CuO}$ chains or $\mathrm{CuO}_{2}$ planes. Therefore, the synergetic effect of high electrical conductivity and higher concentration of oxygen vacancy in YBCO and higher stability of silver particles improves the charge transfer and surface oxygen exchange in the electrode, and as a result, it can promote the electrochemical performance of doped film.
\end{abstract}

Keywords: Electrochemical properties; Silver-doped Yttrium Barium Copper Oxide; Raman spectroscopy; Electrochemical impedance spectroscopy; Cyclic voltammetry

\section{FULL TEXT}


(C) 2021 The Authors. Published by ESG (www.electrochemsci.org). This article is an open access article distributed under the terms and conditions of the Creative Commons Attribution license (http://creativecommons.org/licenses/by/4.0/). 